PROCEEDINGS OF THE

AMERICAN MATHEMATICAL SOCIETY

Volume 137, Number 7, July 2009, Pages 2483-2488

S 0002-9939(09)09791-3

Article electronically published on February 25, 2009

\title{
THE NUMBER OF MINIMAL RIGHT IDEALS OF $\beta G$
}

\author{
YEVHEN ZELENYUK
}

(Communicated by Alexander N. Dranishnikov)

\begin{abstract}
Let $G$ be an infinite Abelian group of cardinality $\kappa$ and let $\beta G$ denote the Stone-Čech compactification of $G$ as a discrete semigroup. We show that $\beta G$ contains $2^{2^{\kappa}}$ many minimal right ideals.
\end{abstract}

Given a discrete semigroup $S$, the operation can be naturally extended to the Stone-Čech compactification $\beta S$ of $S$ making $\beta S$ a compact right topological semigroup with $S$ contained in its topological center. That is, for each $p \in \beta S$, the right translation

$$
\beta S \ni x \mapsto x p \in \beta S
$$

is continuous, and for each $a \in S$, the left translation

$$
\beta S \ni x \mapsto a x \in \beta S
$$

is continuous.

We take the points of $\beta S$ to be the ultrafilters on $S$, the principal ultrafilters being identified with the points of $S$, and $S^{*}=\beta S \backslash S$. The topology of $\beta S$ is generated by taking as a base the subsets of the form

$$
\bar{A}=\{p \in \beta S: A \in p\},
$$

where $A \subseteq S$. For $p, q \in \beta S$, the ultrafilter $p q$ has a base consisting of subsets of the form

$$
\bigcup\left\{x B_{x}: x \in A\right\}
$$

where $A \in p$ and $B_{x} \in q$.

The semigroup $\beta S$ is interesting both for its own sake and for its applications to combinatorial number theory and to topological dynamics. An elementary introduction to $\beta S$ can be found in [5].

As any compact Hausdorff right topological semigroup does, $\beta S$ has a smallest two-sided ideal $K(\beta S)$ which is a disjoint union of minimal right ideals and a disjoint union of minimal left ideals. The intersection of a minimal right ideal and a minimal left ideal is a group, and all these groups are isomorphic. The idempotents of a minimal right (left) ideal form a right (left) zero semigroup, that is, one satisfying the identity $x y=y$ (respectively, $x y=x$ ). See [5, Sections 1.7 and 2.2] for detailed information about this.

Received by the editors February 1, 2008, and, in revised form, September 23, 2008.

2000 Mathematics Subject Classification. Primary 22A15, 22C05; Secondary 22A30, 54H11.

Key words and phrases. Stone-Čech compactification, smallest ideal, minimal right ideal, Abelian group, Bohr compactification.

The author was supported by NRF grant FA2007041200005 and The John Knopfmacher Centre for Applicable Analysis and Number Theory.

(c)2009 American Mathematical Society 2483

Reverts to public domain 28 years from publication 
In [2] and [1], respectively, it was shown that the semigroup $\beta \mathbb{N}$ contains $2^{2^{\omega}}$ minimal left ideals and $2^{2^{\omega}}$ minimal right ideals. The first result has been fully extended to an arbitrary infinite discrete cancellative semigroup $S$ by proving that $\beta S$ contains $2^{2^{|S|}}$ minimal left ideals [5, Theorem 6.42]. However, the problem of counting the minimal right ideals of $\beta S$ turned out to be more difficult. It was only established that for every infinite discrete cancellative semigroup $S, \beta S$ contains at least $2^{2^{\omega}}$ minimal right ideals [5, Corollary 6.41].

The aim of this note is to prove the following result.

Theorem 1. For every infinite discrete Abelian group $G$ of cardinality $\kappa, \beta G$ contains $2^{2^{\kappa}}$ many minimal right ideals.

The proof of Theorem 1 involves some additional concepts.

Recall that the Bohr compactification of a topological group $G$ is a compact topological group $b G$ together with a continuous homomorphism $e: G \rightarrow b G$ such that $e(G)$ is dense in $b G$ and the following universal property holds: For every continuous homomorphism $h: G \rightarrow K$ from $G$ into a compact topological group $K$ there is a continuous homomorphism $h^{b}: b G \rightarrow K$ such that $h=h^{b} \circ e$. In the case where $G$ is a discrete Abelian group, the Bohr compactification can be naturally defined in terms of the Pontrjagin duality as follows. Let $\hat{G}$ be the dual group of $G$ and let $\hat{G}_{d}$ be the group $\hat{G}$ reendowed with the discrete topology. Then $b G$ is the dual group of $\hat{G}_{d}$. The mapping $e: G \rightarrow b G$ is given by $e(x)(\chi)=\chi(x)$, where $x \in G$ and $\chi \in \hat{G}_{d}$. It is injective. (See [4, 26.11 and 26.12].)

We say that filters $\mathcal{F}$ and $\mathcal{G}$ on a set $X$ are incompatible if there are $A \in \mathcal{F}$ and $B \in \mathcal{G}$ such that $A \cap B=\emptyset$. A filter $\mathcal{F}$ on a topological space $X$ is open if $\mathcal{F}$ has a base of open subsets of $X$.

In order to prove Theorem 1, we show the following.

Theorem 2. For every infinite discrete Abelian group $G$ of cardinality $\kappa$, there are $2^{2^{\kappa}}$ many pairwise incompatible open filters on bG converging to zero.

Before proving Theorem 2, let us show how it implies Theorem 1 .

Proof of Theorem 1. Let $\mathcal{T}$ denote the Bohr topology on $G$, that is, the one induced by the mapping $e: G \rightarrow b G$, and let $\mathcal{F}$ be the neighborhood filter of zero of $(G, \mathcal{T})$. By Theorem 2, there are $2^{2^{\kappa}}$ pairwise incompatible open filters on $b G$ converging to zero. Considering the restriction of the filters to $e(G)$, we conclude that there are pairwise incompatible open filters $\mathcal{F}_{\alpha}\left(\alpha<2^{2^{\kappa}}\right)$ on $(G, \mathcal{T})$ converging to zero, that is to say, containing $\mathcal{F}$. Define a closed subset $S$ of $G^{*}$ and for each $\alpha<2^{2^{\kappa}}$, a closed subset $J_{\alpha}$ of $S$ by

$$
S=\bigcap_{U \in \mathcal{F}} \overline{U \backslash\{0\}} \text { and } \quad J_{\alpha}=\bigcap_{U \in \mathcal{F}_{\alpha}} \overline{U \backslash\{0\}}
$$

(Here, $\overline{U \backslash\{0\}}=\{p \in \beta G: U \backslash\{0\} \in p\}$.) Equivalently, $S$ and $J_{\alpha}$ consist of all nonprincipal ultrafilters on $G$ containing $\mathcal{F}$ and $\mathcal{F}_{\alpha}$, respectively. We claim that $S$ is a subsemigroup of $G^{*}$ and for each $\alpha<2^{2^{\kappa}}, J_{\alpha}$ is a right ideal of $S$.

To see that $S$ is a subsemigroup, let $p, q \in S$. We have to show that for every $U \in \mathcal{F}$, one has $U \backslash\{0\} \in p+q$. Without loss of generality one may assume that $U$ is open. For every $x \in U \backslash\{0\}$, choose $V_{x} \in \mathcal{F}$ such that $x+V_{x} \subseteq U \backslash\{0\}$. Then $U \backslash\{0\}=\bigcup_{x \in U \backslash\{0\}}\left(x+V_{x}\right)$. Since $U \backslash\{0\} \in p$ and $V_{x} \in q$, we obtain that 
$U \backslash\{0\} \in p+q$. The same argument shows that for every $p \in R_{\alpha}, q \in S$ and $U \in \mathcal{F}_{\alpha}$, one has $U \backslash\{0\} \in p+q$, which witnesses that $J_{\alpha}$ is a right ideal.

Since the filters $\mathcal{F}_{\alpha}$ are pairwise incompatible, the right ideals $J_{\alpha}$ are pairwise disjoint. Taking a minimal right ideal in each $J_{\alpha}$, we obtain that there are $2^{2^{\kappa}}$ minimal right ideals of $S$. Furthermore, since $(G, \mathcal{T})$ is a subgroup of a compact topological group, $S$ contains all the idempotents of $G^{*}$ [6, Lemma 3], in particular, the idempotents of $K(\beta G)$. (Note that $K(\beta G) \subseteq G^{*}$, because $G^{*}$ is an ideal of $\beta G$.) Consequently, $S \cap K(\beta G) \neq \emptyset$. But then, by [5, Theorem 1.65],

$$
K(S)=K(\beta G) \cap S .
$$

It follows from this that every minimal right ideal $R$ of $S$ is contained in a minimal right ideal $R^{\prime}$ of $\beta G$, and the correspondence $R \mapsto R^{\prime}$ is injective.

Indeed, $K(\beta G)$ is a union of minimal right ideals, so there is a minimal right ideal $R^{\prime}$ of $\beta G$ such that $R \cap R^{\prime} \neq \emptyset$. Then $R \cap R^{\prime}$ is a right ideal of $S$ contained in $R$. Consequently, $R \cap R^{\prime}=R$, as $R$ is minimal, and so $R \subseteq R^{\prime}$. Since minimal right ideals are disjoint, such an $R^{\prime}$ is unique. To see that the correspondence $R \mapsto R^{\prime}$ is injective, let $R_{1}$ and $R_{2}$ be minimal right ideals of $S$ and assume that $R_{1}^{\prime}=R_{2}^{\prime}$. Pick any minimal left ideal $L$ of $S$ and let $p_{1}$ and $p_{2}$ be the identities of the groups $R_{1} \cap L$ and $R_{2} \cap L$, respectively. Being idempotents of $L, p_{1}$ and $p_{2}$ belong to the same left zero semigroup, so $p_{1}+p_{2}=p_{1}$, and being idempotents of $R_{1}^{\prime}=R_{2}^{\prime}$, they belong to the same right zero semigroup, so $p_{1}+p_{2}=p_{2}$. Hence, $p_{1}=p_{2}$, and consequently, $R_{1}=R_{2}$. (In fact, this correspondence is bijective, since $S$ contains all the idempotents of $K(\beta G)$.)

It follows that the number of minimal right ideals of $\beta G$ is greater than or equal to that of $S$, and consequently, it is $2^{2^{\kappa}}$.

To prove Theorem 2 we need three lemmas. The first of them is an elementary fact on infinite Abelian groups.

Lemma 1. Let $G$ be an infinite Abelian group of cardinality $\kappa$. Then $G$ admits a homomorphism onto one of the following groups:

(1) $\mathbb{Z}, \bigoplus_{\omega} \mathbb{Z}(p), \mathbb{Z}\left(p^{\infty}\right)$ and $\bigoplus_{p \in Q} \mathbb{Z}(p)$ if $\kappa=\omega$,

(2) $\bigoplus_{\kappa} \mathbb{Z}(p)$ and $\bigoplus_{\kappa} \mathbb{Z}\left(p^{\infty}\right)$ if $\kappa>\omega$ and $\operatorname{cf}(\kappa)>\omega$,

(3) $\bigoplus_{\kappa} \mathbb{Z}(p), \bigoplus_{\kappa} \mathbb{Z}\left(p^{\infty}\right), \bigoplus_{p \in Q} \bigoplus_{\kappa_{p}} \mathbb{Z}(p)$ and $\bigoplus_{p \in Q} \bigoplus_{\kappa_{p}} \mathbb{Z}\left(p^{\infty}\right)$ if $\kappa>\omega$ and $\operatorname{cf}(\kappa)=\omega$.

Here, $p$ is a prime number and $Q$ is an infinite subset of the primes. The symbols $\mathbb{Z}, \mathbb{Z}(p)$ and $\mathbb{Z}\left(p^{\infty}\right)$ denote the infinite cyclic group, the cyclic group of order $p$, and the quasi-cyclic group, respectively. The symbol $\operatorname{cf}(\kappa)$ denotes the cofinality of $\kappa$. If $\kappa>\omega$ and $\operatorname{cf}(\kappa)=\omega,\left(\kappa_{p}\right)_{p \in Q}$ is an infinite increasing sequence of uncountable cardinals cofinal in $\kappa$, that is, $\sup _{p \in Q} \kappa_{p}=\kappa$.

Proof. If $G$ is finitely generated, then $\kappa=\omega$ and $G$ admits a homomorphism onto $\mathbb{Z}$. Therefore, one may assume that $G$ is not finitely generated. We first prove that $G$ admits a homomorphism onto a periodic group of cardinality $\kappa$.

Let $\left\{a_{i}: i \in I\right\}$ be a maximal independent subset of $G$ and let $A=\left\langle a_{i}: i \in I\right\rangle$ be the subgroup generated by $\left\{a_{i}: i \in I\right\}$. Then $A=\bigoplus_{i \in I}\left\langle a_{i}\right\rangle$, and for every nonzero $g \in G$, one has $\langle g\rangle \cap A \neq\{0\}$, so $G / A$ is periodic. If $|G / A|=\kappa$, we are done. Suppose that $|G / A|<\kappa$. Then $|A|=\kappa$ and $|I|=\kappa$, because $G$ is not finitely generated. We show that there is a subgroup $H$ of $G$ and a subset $I_{1} \subseteq I$ with $\left|I_{1}\right|=\kappa$ such that $G=H \oplus \bigoplus_{i \in I_{1}}\left\langle a_{i}\right\rangle$. 
To this end, choose a complete set $S$ for representatives of the cosets of $A$ in $G$, and let $H_{0}=\langle S\rangle \cap A$. Define $I_{0} \subset I$ by

$$
I_{0}=\left\{i \in I: x(i) \neq 0 \text { for some } x \in H_{0}\right\} \text {, }
$$

where $x(i)$ is the $i$-th coordinate of $x$, and put $I_{1}=I \backslash I_{0}$. If $G / A$ is finite, $I_{0}$ is finite as well. If $G / A$ is infinite, $\left|I_{0}\right| \leq|G / A|$, because $|\langle S\rangle|=|G / A|$ and then $\left|H_{0}\right| \leq|G / A|$. In any case, $\left|I_{0}\right|<\kappa$, and consequently $\left|I_{1}\right|=\kappa$. Let

$$
A_{0}=\left\langle a_{i}: i \in I_{0}\right\rangle, \quad A_{1}=\left\langle a_{i}: i \in I_{1}\right\rangle \quad \text { and } \quad H=\left\langle S \cup A_{0}\right\rangle .
$$

We claim that $G=H \oplus A_{1}$. Indeed, since $G=\left\langle S \cup A_{0} \cup A_{1}\right\rangle$, one has $H+A_{1}=G$. To see that $H \cap A_{1}=\{0\}$, let $g \in H \cap A_{1}$. Then $g=d+c_{0}=c_{1}$ for some $d \in\langle S\rangle$, $c_{0} \in A_{0}$ and $c_{1} \in A_{1}$. Consequently, $d=-c_{0}+c_{1} \in A$. But then $d \in H_{0} \subseteq A_{0}$. Hence, $c_{1}=0$, and $g=0$.

Having established that $G=H \oplus \bigoplus_{i \in I_{1}}\left\langle a_{i}\right\rangle$, we obtain that $G$ admits a homomorphism onto $\bigoplus_{i \in I_{1}}\left\langle a_{i}\right\rangle$, and so onto a periodic group of cardinality $\kappa$.

Now let $G$ be a $p$-group. Then there is a so-called basic subgroup $B$ of $G$ (see [3. Theorem 32.3]). We have that $B$ is a direct sum of cyclic groups, say $B=\bigoplus_{j \in J}\left\langle b_{j}\right\rangle$, and $G / B$ is divisible, that is, isomorphic to $\bigoplus_{\lambda} \mathbb{Z}\left(p^{\infty}\right)$, where $0 \leq \lambda \leq \kappa$. Suppose that $|G / B|=\kappa$. Then $\lambda>0$, and $\lambda=\kappa$ if $\kappa>\omega$. It follows that $G$ admits a homomorphism onto $\mathbb{Z}\left(p^{\infty}\right)$ if $\kappa=\omega$, and onto $\bigoplus_{\kappa} \mathbb{Z}\left(p^{\infty}\right)$ if $\kappa>\omega$. Now suppose that $|G / B|<\kappa$. Then $|B|=\kappa$, and consequently $|J|=\kappa$. It follows that $G=C \oplus \bigoplus_{j \in J_{1}}\left\langle b_{j}\right\rangle$ for some subgroup $C$ of $G$ and a subset $J_{1} \subset J$ with $\left|J_{1}\right|=\kappa$ (see the first part of the proof). Hence, $G$ admits a homomorphism onto $\bigoplus_{j \in J_{1}}\left\langle b_{j}\right\rangle$, and so onto $\bigoplus_{\kappa} \mathbb{Z}(p)$.

Finally, let $G$ be periodic. Then $G=\bigoplus_{p \in M} G_{p}$, where $M$ is the set of all primes $p$ such that the $p$-primary component $G_{p}$ of $G$ is nontrivial. If $\left|G_{p}\right|=\kappa$ for some $p \in M$, we are done, because then $G$ admits a homomorphism onto $G_{p}$, a $p$-group of cardinality $\kappa$. Suppose that $\left|G_{p}\right|<\kappa$ for each $p \in M$. Then $M$ is infinite and $\operatorname{cf}(\kappa)=\omega$. If $\kappa=\omega$, all $G_{p}$ are finite, and so $G$ admits a homomorphism onto $\bigoplus_{p \in M} \mathbb{Z}(p)$. Suppose that $\kappa>\omega$. For each $p \in M$, put $\kappa_{p}=\left|G_{p}\right|$. Clearly $\sup _{p \in M} \kappa_{p}=\kappa$. Choose an infinite subset $N \subseteq M$ such that $\left(\kappa_{p}\right)_{p \in N}$ is an increasing sequence of uncountable cardinals cofinal in $\kappa$. By the previous paragraph, for each $p \in N, G_{p}$ admits a homomorphism onto a group $K_{p}$ of cardinality $\kappa_{p}$ which is isomorphic to $\bigoplus_{\kappa_{p}} \mathbb{Z}(p)$ or $\bigoplus_{\kappa_{p}} \mathbb{Z}\left(p^{\infty}\right)$. It follows that there is an infinite subset $Q \subseteq N$ such that either $K_{p}$ is isomorphic to $\bigoplus_{\kappa_{p}} \mathbb{Z}(p)$ for all $p \in Q$ or $K_{p}$ is isomorphic to $\bigoplus_{\kappa_{p}} \mathbb{Z}\left(p^{\infty}\right)$ for all $p \in Q$. Then the group $K=\bigoplus_{p \in Q} K_{p}$ is isomorphic to $\bigoplus_{p \in Q} \bigoplus_{\kappa_{p}} \mathbb{Z}(p)$ or $\bigoplus_{p \in Q} \bigoplus_{\kappa_{p}} \mathbb{Z}\left(p^{\infty}\right),|K|=\kappa$, and $G$ admits a homomorphism onto $K$.

Now, using Lemma 1 and the Pontrjagin duality, we prove the following statement on $b G$.

Lemma 2. For every infinite discrete Abelian group $G$ of cardinality $\kappa, b G$ admits a continuous homomorphism onto $\prod_{2^{\kappa}} \mathbb{T}$ or $\prod_{2^{\kappa}} \mathbb{Z}(p)$.

Here, $\mathbb{T}$ is the circle group, and both products $\prod_{2^{\kappa}} \mathbb{T}$ and $\prod_{2^{\kappa}} \mathbb{Z}(p)$ are endowed with the product topology.

Proof. The dual groups of continuous homomorphic images of $b G$ are the subgroups of $\hat{G}_{d}$, and the dual groups of homomorphic images of $G$ are the closed subgroups 
of $\hat{G}$ (see [4, Theorems 23.25 and 24.8]). The dual groups of $\prod_{2^{\kappa}} \mathbb{T}$ and $\prod_{2^{\kappa}} \mathbb{Z}(p)$ are $\bigoplus_{2^{\kappa}} \mathbb{Z}$ and $\bigoplus_{2^{\kappa}} \mathbb{Z}(p)$, respectively. Consequently, in order to prove the lemma, it suffices to show that $G$ admits a homomorphism onto a group whose dual group contains an isomorphic copy of $\bigoplus_{2^{\kappa}} \mathbb{Z}$ or $\bigoplus_{2^{\kappa}} \mathbb{Z}(p)$. We distinguish between two cases.

Case 1. $\kappa=\omega$. Then $G$ admits a homomorphism onto one of the following groups: $\mathbb{Z}, \bigoplus_{\omega} \mathbb{Z}(p), \mathbb{Z}\left(p^{\infty}\right)$ and $\bigoplus_{p \in Q} \mathbb{Z}(p)$. Their dual groups are $\mathbb{T}, \prod_{\omega} \mathbb{Z}(p), \mathbb{Z}_{p}$ and $\prod_{p \in Q} \mathbb{Z}(p)$, respectively. (Here, $\mathbb{Z}_{p}$ is the group of $p$-adic integers.) The second group is algebraically isomorphic to $\bigoplus_{2 \omega} \mathbb{Z}(p)$. The others contain torsion-free subgroups of cardinality $2^{\omega}$ and so contain an isomorphic copy of $\bigoplus_{2^{\omega}} \mathbb{Z}$.

Case 2. $\kappa>\omega$. Then $G$ admits a homomorphism onto one of the following groups: $\bigoplus_{\kappa} \mathbb{Z}(p), \bigoplus_{\kappa} \mathbb{Z}\left(p^{\infty}\right), \bigoplus_{p \in Q} \bigoplus_{\kappa_{p}} \mathbb{Z}(p)$ and $\bigoplus_{p \in Q} \bigoplus_{\kappa_{p}} \mathbb{Z}\left(p^{\infty}\right)$ (the two latter groups appear if $\operatorname{cf}(\kappa)=\omega)$. Their dual groups are $\prod_{\kappa} \mathbb{Z}(p), \prod_{\kappa} \mathbb{Z}_{p}, \prod_{p \in Q} \prod_{\kappa_{p}} \mathbb{Z}(p)$ and $\prod_{p \in Q} \prod_{\kappa_{p}} \mathbb{Z}_{p}$, respectively. The first group is algebraically isomorphic to $\bigoplus_{2^{\kappa}} \mathbb{Z}(p)$. The others contain torsion-free subgroups of cardinality $2^{\kappa}$ and so contain an isomorphic copy of $\bigoplus_{2^{\kappa}} \mathbb{Z}$

The third lemma deals with products of topological spaces.

Lemma 3. Let $\kappa$ be an infinite cardinal. For each $\alpha<\kappa$, let $X_{\alpha}$ be a space having at least two disjoint nonempty open sets, and let $X=\prod_{\alpha<\kappa} X_{\alpha}$. Then there are at least $2^{\kappa}$ many pairwise incompatible open filters on $X$ converging to the same point.

The proof of Lemma 3 involves the notion of an extremally disconnected space, that is, a space in which the closures of disjoint open sets are disjoint. Notice that if each factor in an infinite product $X=\prod_{n<\omega} X_{n}$ has at least two disjoint nonempty open sets, then $X$ is not extremally disconnected. Indeed, for each $n<\omega$, let $U_{n}$ and $V_{n}$ be disjoint nonempty open subsets of $X_{n}$, let $x_{n} \in V_{n}$, and let $x=\left(x_{n}\right)_{n<\omega} \in X$. For every $m<\omega$, define an open subset $W_{m}=\prod_{n<\omega} W_{m, n} \subset X$ by

$$
W_{m, n}= \begin{cases}U_{n} & \text { if } n=m, \\ V_{n} & \text { if } n<m, \\ X_{n} & \text { if } n>m .\end{cases}
$$

It follows that $U=\bigcup_{m<\omega} W_{2 m}$ and $V=\bigcup_{m<\omega} W_{2 m+1}$ are disjoint open subsets of $X$ with $x \in c \ell U \cap c \ell V$.

Proof of Lemma 3. Let $L$ be the set of limit ordinals $<\kappa$ including 0 . Then

$$
X=\prod_{\alpha \in L} \prod_{n<\omega} X_{\alpha+n},
$$

$|L|=\kappa$, and for each $\alpha \in L, \prod_{n<\omega} X_{\alpha+n}$ is not extremally disconnected. Therefore, one may suppose that each $X_{\alpha}$ in the product $X=\prod_{\alpha<\kappa} X_{\alpha}$ is not extremally disconnected, so there are disjoint open subsets $U_{\alpha}, V_{\alpha} \subset X_{\alpha}$ and $x_{\alpha} \in X_{\alpha}$ such that $x_{\alpha} \in c \ell U_{\alpha} \cap c \ell V_{\alpha}$. For every $Y=\left(Y_{\alpha}\right)_{\alpha<\kappa} \in \prod_{\alpha<\kappa}\left\{U_{\alpha}, V_{\alpha}\right\}$, define the filter $\mathcal{F}(Y)$ on $X$ by declaring as a base the subsets of the form $\prod_{\alpha<\kappa} Z_{\alpha}$, where

(i) for each $\alpha<\kappa, Z_{\alpha}$ is a nonempty open subset of $X_{\alpha}$,

(ii) for all but finitely many $\alpha<\kappa, Z_{\alpha}=X_{\alpha}$, and

(iii) if $Z_{\alpha} \neq X_{\alpha}$, then $Z_{\alpha}=Y_{\alpha} \cap W$ for some neighborhood $W$ of $x_{\alpha} \in X_{\alpha}$. 
Then $\mathcal{F}(Y)$, where $Y \in \prod_{\alpha<\kappa}\left\{U_{\alpha}, V_{\alpha}\right\}$, are pairwise incompatible open filters on $X$ converging to $x=\left(x_{\alpha}\right)_{\alpha<\kappa}$.

Now we are in a position to prove Theorem 2 .

Proof of Theorem 2. By Lemma 2, there is a continuous surjective homomorphism $f: b G \rightarrow K$, where $K$ is $\prod_{2^{\kappa}} \mathbb{T}$ or $\prod_{2^{\kappa}} \mathbb{Z}(p)$. By Lemma 3, there are pairwise incompatible open filters $\mathcal{F}_{\alpha}\left(\alpha<2^{2^{\kappa}}\right)$ on $K$ converging to zero. For each $\alpha<2^{2^{\kappa}}$, let $\mathcal{H}_{\alpha}$ be the filter on $b G$ with a base consisting of subsets of the form $f^{-1}(A) \cap U$, where $A \in \mathcal{F}_{\alpha}$ and $U$ runs over neighborhoods of zero. Then $\mathcal{H}_{\alpha}\left(\alpha<2^{2^{\kappa}}\right)$ are pairwise incompatible open filters on $b G$ converging to zero.

\section{ACKnowledgement}

The author would like to thank the referee for a careful reading of the paper and useful comments.

\section{REFERENCES}

1. J. Baker and P. Milnes, The ideal structure of the Stone-Čech compactification of a group, Math. Proc. Cambridge Philos. Soc. 82 (1977), 401-409. MR0460516 (57:509)

2. C. Chou, On a geometric property of the set of invariant means on a group, Proc. Amer. Math. Soc. 30 (1971), 296-302. MR0283584 (44:815)

3. L. Fuchs, Infinite Abelian Groups. I, Academic Press, New York and London, 1970. MR0255673 $(41: 333)$

4. E. Hewitt, K. Ross, Abstract harmonic analysis. I, Springer-Verlag, Berlin and New York, 1979. MR.551496 (81k:43001)

5. N. Hindman and D. Strauss, Algebra in the Stone-Čech compactification, de Gruyter, Berlin, 1998. MR 1642231 (99j:54001)

6. Y. Zelenyuk, On the ultrafilter semigroup of a topological group, Semigroup Forum 73 (2006), 301-307. MR2280826 (2007i:22004)

School of Mathematics, University of the Witwatersrand, Private Bag 3, Wits 2050, South AFricA

E-mail address: yevhen.zelenyuk@wits.ac.za 